\title{
Populations and Excavations; Strata and Data: A linguist Muses upon Anthropology and Archaeology
}

\author{
Tully J Thibeau* \\ University of Montana, Missoula, USA \\ *Corresponding author: Tully J Thibeau, University of Montana, Missoula, USA
}

\begin{abstract}
This paper discusses a small but significant bond that Anthropology and Archaeology share with Linguistics. The discussion is organized around the notion that Anthropology defines human languages as synchronic (i.e., together-time) in opposition to Archaeology, which defines them as diachronic (i.e., across-time). Regarding a Linguistic definition, my discussion of it considers them a matter of optimal design (ekchronic, or outside-time), and, as a result, thinks of them neither communicatively, in terms of ethnography of speaking, nor philologically, in terms of writings of antiquity. However, the modern definition of a science of human languages requires that they be treated as quantities that are calibrated in order to become metricizeable (i.e., not merely so many bits and pieces that materialize). Admittedly, they look that way, but the difference is what appears within chance and beyond it, more like a conceptual metaphor of material culture from statistics [1]. I mainly resolve that labeling linguistic material in this style fails to explain human languages in personal interaction or cognition; they alone have 'structure' in an arithmetic construal, but the extent to which human languages interface with either spoken or written words cannot be (very easily, if at all) fathomed intra-disciplinarily.
\end{abstract}

\section{Introductory Remarks}

Linguistics has been regarded by some as a subfield of Anthropology (on a plane of synchrony) and as a distant relative to Archaeology (on a plane of diachrony), chiefly in terms of Philology, though some in a few places may deem distinction between them as simplistic [2]. Despite which disciplinary compartment a science of language belongs in, language scientists will surely experience a dynamic tension existing between unpredictable and predictable gestures which are represented behaviorally and/or cognitively in naturally occurring human languages.

For example of such gestures, the unpredictable ones comprise the set of idiosyncratic features that differ from language to language: The English word for ten is, in Turkish, the word on, and Eng. den (meaning 'animal's lair') is Trk. in (similar meaning): That is, in human languages, random sounds (vocal images) symbolize equivalent interpretations (mental images), or glosses; moreover, within an individual language variety (e.g., English and Turkish), contrastive sounds often differentiate one mental image from another (Eng. $t$-en v. $d$-en, Trk. $o-n$ v. $i-n$ ). The pairs of forms, or vocal images, in English and Turkish are unpredictable yet contrast in their meaning, and words and sounds constituting words must hence be stored in long-term memory 'lexically' [3].
However, not all sounds or words (i.e., meaningful units of sounds) need be memorized in "the lexicon" but instead are relatively predictable gestures with reasonably regular meanings. While English $t$ and $d$ are gestures in contrastive distribution (a minimal pair) at a level of sound (as was rendered above), they are not so at a level of 'minimal unit' of meaning: For instance, the English words tack and tag exhibit nominal properties (e.g., they can be pluralized as nouns) as well as verbal properties (e.g., they can form past-tenses as verbs); moreover, the form tack receives the past-tense suffix $t$ (in speech), yet the form tag receives the past-tense suffix $d$.

English at one level of sound (phonemics) must separate $t$ and $d$ as different (in word-meaning) but the same (phonetics) at another level (suffix, morphology below the level of word-meaning). These morphological $\mathrm{t}$ and d gestures in English are predictable ones, which are stored as rules [3].

As an over-simplified view of storage of language, the unpredictable gestures get saved lexically, or as memory-based items, and the predictable ones get saved as a part of a grammar, or rule-based items [4]. This over-simplification concerns cognition, namely knowledge, on which language skill, or behavior, is based; 
language data (i.e., behavior) are exposed to a set of two cognitive procedures, one being passably concrete (unanalyzed data), the other tolerably abstract (analyzed data). These routes bid to describe a developmental path and may be easily likened to language functions as imitative wholes deposited as lexical material (early development) but riven into language forms, mapping onto functions (later development). Karmiloff-Smith [5] equates these stages as implicit mental representations that are obscured (hence unanalyzable) to learners and then gradually more explicit mental representations that are increasingly scannable (i.e., analyzable) for learners; 'lexical' items may become grammaticized. This conception of development may reveal more about cognition than about language, however.

\section{Revealing More About Language, or What You See is Not What You Get}

As an illustration, please take for example those gestures that are unpredictable and therefore usually associated with a characteristic similar to the arbitrariness of linguistic sign [6]. That is to say, human languages link (map) vocal images to mental images in overly direct one-to-one ways: An early developmental stage is called "nominal realism" [7,8] and 'underextension' by developmental psycholinguists: When children commence to develop their vocabularies, they map a vocal image onto a mental image, one taken from the physical properties (or the raw data) of the discovery-event [9] as a "haphazard" exemplar [10], not realizing that a word in general represents a set of objects. If one of the physical properties of that random example changes (e.g., color, size), then a different word (the child might insist) must be required to denote that particular object (e.g., the vocal image boots may not be accepted by a child learning English if the 'boots' are of dissimilar color and/or size than when the child initially perceived a first mental image of boots). The opposite phenomenon of 'underextension' is 'overextension' (e.g., a child using the vocal image ball for all mental images round in shape). Some perceptual properties, like roundness, receive a suspected universal salience in terms of children's overextensions [11] and suggest that meanings of words are organized (at least in part) as componential analyses, broken down into semantic primitives assigned distinctive features with \pm oppositions [12].

From here, matters become awkward in two different ways, with respect to Anthropolgy and Archaeology, for the study of culture according to this mode may be discerned as structural, thus insuffiently explanatory [13], and the study of the written forms from antiquity coherent with this system may be avowed in an alike way: "When numbers dominate the Word, this same false hope of becoming respectable through quantification..." [2]. Many of the problems that are related to language are left to applied linguists [14] although, to linguists, language-related problems, while not appraised as such by native speakers (either past or present), are yet resolvable by means of simplifications of such quantifications.
Quantifications in terms of binary oppositions harnassed to distinctive features were popularized as a presence or an absence of a precise physical distinctive feature (e.g., voicing) by Saussure; his "algebraic notation" allowed an application (in retrograde) for historical-linguistic purposes, that is, predicting (rather than reconstructing) that Hittite would follow rules (at a level of sound) of Indo-European (IE), based on the comparative technique of the $19^{\text {th }}$ century neogrammarians [15]. It was in 1927, fourteen years after Saussure's death, that Kurylowicz indicated that Hittite utilized a written gesture matching cognate IE forms that Saussure conjectured, using his method of arithmetical analyses of 'phonemes' and the way that they "patterned" with morphological units [16]; for good or ill, at this point, any subject matter experts must come to terms with '-emic' and '-etic' levels of representation.

Strictly speaking, Saussure does not, in his work on IE "sonant coeffiecents" (laryngeals), define what a phoneme is; rather, he uses the term with the implicit understanding of his readers, that one is empty of a physical gesture (i.e., 'phonetic' content) but serves as an abstraction away from a vocal quality [17], characterized by some principal rudiments of sound (i.e., 'phonemic' content). While styled as not fitting into any structuralist school of linguistics, Kurylowicz presumed no less than two strata for human languages (e.g., 'langue' and 'paroles'), an "abstract" stratum composed of meanings, or morphemes (i.e., "unstructutured," or arbitrary), "only represented" (yet "not composed") by fundamental -emic "constituents," incorporating another materialistic ${ }^{1}$ stratum of directly observable data [18]. At stake is the idea that a unit of language, some gesture (e.g., vocal image) mapping onto a mental one, is either a static form (which may in fact vary according to its position in relation to other units) or a dynamic one (which may in fact vary according to a procedure working on one basic shape). Rather than take issue with any mechanism that may/may not be involved (i.e., remain agnostic), focus should instead be placed on the materials utilized when linking sound and meaning as one; human languages are, after all, to some degree arbitrary in their behavior, and no human being, regardless of expertise, directly observes what is happening cognitively, where mapping occurs. However, if all parts of human languages were, in their behavior, happening wholly by chance, then no human being could project what would be considered as normative by the community.

\section{A Brief Post-Structural Interlude}

The part of human languages, where predictions based on community norms are formed, is the grammar. A human grammar may be a set of rule-based mental representations or may be a set of exemplar-based mental representations which provide cognition with repetitive patterns that resemble rule-based mental representations yet are induced only from behavior (or the data). This choice of one view of human grammar over another view might appear to be an option set forth in terms of political philosophy: Does humanity merely "recover" meanings from its past or are they "something we create" [19], in the parlance of linguists, generate?

${ }^{1}$ Bloomfield 1933 believed that the attribute "mechanistic" was optimal over "materialistic." 


\section{Specifications for a 'Linguistic' Economy}

For linguists, concerns of this partisan or ethical hue are not as great as language design, the extent to which how deftly a language's arbitrary exemplifications stand in constant parallel with its (proportional) logicalities: That is to say (all things being equal), a solitary sound (be it at a phonemic or phonetic stratum) does not map onto a meaning unit; all meaning units compose either a lexicon (randomly) or a grammar (calculatingly). An English illustration discloses that 'alveolar fricatives' that differ in 'voicing' features (symbolically $s$ and $z$ ) represent no meanings in and of themselves (besides onomatopoetically ${ }^{2}$ ); however, they express a meaning contrast between cease /sis/and seize /siz/ in wordfinal position (i.e., contrastive distribution) and thus are regarded at the level of sound as (unpredictably) phonemic. Phonologically phonemes, they (still symbolically $s$ and $z$ ) function as a suffix (i.e., morpheme) to these lexical items and signal the present tense of a $3^{\text {rd }}$ person singular subject (it ceases /sis-iz/ v. it seizes /siz$i z /$ ), and this suffix varies proportionally with the same subject (it stops /stap-s/ v. it grabs /græb-z/); at a morphological level, s and $\mathrm{z}$ are no longer regarded as contrastive but as complementary (i.e., this suffix varies according to phonemic properties of a wordfinal phonological segment). These morphological variations (or allomorphs) might be considered static units /-iz -s - -z/ (i.e., arranged in relation to a lexical word) or dynamic units whereby /-izz -s $\sim-\mathbf{z} /$ are derived from a single would-be 'archiphoneme' /-Z/ by means of a putatively cognitive process; regardless of how this linguistic phenomenon is optimally described, processes could nonetheless never be directly observed cognitively, but they could be considered as such, yet only historically [20].

This discussion now is not so much concentrated on an optimal description of design of human languages but redirected toward the degree (moving properly parallel or perpendicular to) that a professed synchronic process in the mind echoes an attested or inferred diachronic process. Optimal language design is centered on phraseology such as parsimony, elegance and simplicity, in the sense that Occam's Razor upholds a minimal account for complex affairs: An example of such plainness in describing human language includes the aforesaid 'archiphoneme,' which neutralizes a contrast between two phonemes (Trk. at 'horse' v. at 'name', seeming homonyms, become at-i 'its horse' v. ad-i 'its name', a voicing difference neutralized in word-final position).

In Turkish, $t$ and $d$ are phonemes in word-initial position (e.g., $t$-ek 'single' v. $d$-ek 'until'), yet their voicing distinctions $( \pm)$, which make them contrastive, are rendered to be no longer relevant in word-final position. Trubetzkoy [21] created the term 'archiphoneme' for a kind of cross-linguistic phenomena revealing an added "mark" (e.g., [+ voice]) only when a segment (e.g., a stop) is positioned in a given linguistic context (in the prior specimen, between vowels). The implication that the proposal of an archiphoneme entails is, in part, that it is only practicable to propose one if linguists understand a language's phonological structure (phonemic/phonetic) as well as its morphological structure; the other part of the implication is that the phonology of $\mathrm{X}$ is, with respect to at least one of its facets, contingent on the morphology of $X$ [22]. In specific linguistic contexts, segments of phonetics could be abstracted by pleading phonemics.

In labeling the synchronic state of language $\mathrm{X}$ (for example, English and an optimal way to describe its morphological variants in present tense 3rd-person singular properties of a subject, namely, /-iz - -s $\sim-\mathbf{z} /$ ), the term 'archiphoneme' might not be a suitable label because forms of English verbs remain constant while a soi-disant suffix /-Z/ exhibits 'archiphonemic' behavior. In this case of variation, the 'subject-verb agreement' morphology is phonologically conditioned, based on the synchronic state of the phonological structure that define English word-boundaries; suffixes (below the level of word, but not of meaning) undergo variations based on the boundary that defines word-final. Whether the solitary meaning of the suffix should be preserved as /-Z/ or the directly observable phonetic realizations should be reflected as /-iz $\sim-\mathbf{s} \sim-\mathbf{z} /$ is a matter that designers must muse upon. As two language-change incidents over time, however, it is observed that archiphonemic neutralizations may not be easily subsumed as morphophonemic alternations (a.k.a., phonologically-conditioned morphemes): Modes of accosting these two types of changes may be listed as 'archiphonemic vs. phonemic' or 'typical' (i.e., circumventing any differences between the two procedures, thus rendering them all as 'sound changes'); in particular instances, sound-change over time proceeds according to a 'phonemic' principle and, in further instances, according to a 'archiphonemic' principle [23]. Usage of phraseology such as principle suggests that diachronic processes present language design with glimpses into mental processes.

\section{Outlining Rules Through a Ranking Thereof}

In observing any principles of sound-changes at wordboundaries involving morphemes, no clear-cut claims have been made pertaining to an adequate standard for rendering more simple the sets formed by language $\mathrm{X}$. Various diachronic descriptions of ostensible proto-languages, however, refer to alleged synchronic descriptions of how those past systems must have operated. As for a System, I hazard one to be a set of principles, and a principle to be a way of proceeding (in this instance, using language X intelligibly, in terms of its perception/production in speech).

Not only must one include a means of storing samplings from experience into unpredictable and predictable repositories (the latter into paradigmatic form), but also one must institute a sequence of variations among segments such that those patterns are deposited into a predictable repository. In other words, this system is 'trained' to be capable of stockpiling descriptive material apropos of language $\mathrm{X}$ and to be capable of prioritizing those materials, based on ranges of variability.

As an illustration, recall the aforementioned variations among /-iz - -s - -z/ where the form /-iz/ shares the narrowest distribution (linear array), directly adjacent to the English sound segments

${ }^{2}$ Some place between Bloomfield's remarks (1933, p. 6) regarding a "bow-wow" theory and a "ding-dong" theory.

Citation: Tully J Thibeau. Populations and Excavations; Strata and Data: A linguist Muses upon Anthropology and Archaeology. J Anthro 
/s- $\sim \mathbf{Z}^{-} \sim \int-\sim \mathbf{z}-\sim \mathbf{t}-\sim \mathbf{d z}-/$ at the ends of words (often referred to sibilants); conversely, unlike /-iz/,the variant /-z/ shares the widest distribution (immediately after any segment), and /-s/ immediately after any segment specified as [-voice]. The variant /-z/, because it is allotted the widest distribution, becomes the default marker of 3rd-person subject present-tense agreement (the 'elsewhere' condition), providing a system simplicity in calculating the motor instructions for producing/perceiving all relevant forms (i.e., /-iz $\mathbf{s} \sim-\mathbf{z} /$ ) synchronically [24].

Perhaps a better way of defining simplicity at the systematic level (e.g., grammar rules), the output of this morphophonemic alternation is the unmarked form, which joins a 'basic' form (or root or stem of a verb) with a suffix that signals a third-person singular present-tense subject. If that root or stem of a verb ends in a sound segment with a [-voice] property, then the suffix also becomes so marked (i.e., unmarked /-z/form proceeds to the marked /-s/ form, harmonizing in [-voice] features). Furthermore, with regard to the segments /s- $\mathbf{z}-\sim \int-\sim \mathbf{z}_{-} \sim \mathbf{t} \int-\sim \mathbf{d} \mathbf{3}-/$, the sibilants, a more specific environment is conditioned, wherein the root or stem of a verb ends in sound segments with combined primary and secondary obstruction [+consonantal] features: The primary one involves lifting the front edge of the tongue towards the alveolar ridge or teeth (i.e., [+coronal]); the secondary one involves the constriction of the air flow being aimed toward either primary place of articulation in order to augment the airflow of the sound segment (i.e., [+strident]), a property found only among [+continuant] [+obstruent] segments, or fricatives and affricates [25]. That is to say, the simpler the property that enters into the input (basic + suffix), the least marked form that exits the output (verb $+z$ ); once properties are added (either [-voice] or [+coronal] [+strident]), marked forms ensue. An elsewhere condition is a default unmarked signal (third-person singular present-tense subject) unless lower-level properties intercede the unmarked forms to generate marked forms by 'rule,' or order. This construal presumes that only applicable features or properties are indispensable for triggering marked forms, restricted to [+coronal] [+strident] (or [-voice]) rather than every single one that distinguishes each form: "The fewer features mentioned in the description, the greater its economy... the reason for establishing an order is made explicit ... [an aftereffect] of the proposed criterion of simplicity" [24]. In this theory of a grammar, what we cannot directly observe (a mental process) is inferred from what we observe directly, synchronic behavior. Such inferences are equally true for various diachronic processes as well. The relationship between the sound changes described as Grimm's and Verner's laws illustrate a simplicity criterion with a goal toward ordering these two sound-change regularities by combining attested forms from a Proto-Germanic language and forms inferred there from.

The affiliation of these regular sound-changes is a sequence of two ordered regulations in which one must have preceded the other and, as a consequence, the demands of the predecessor ruling widened the 'natural class' (i.e., a set of sounds affected by the first instruction) of the segments that the successor ruling applied to. Grimm's Law notably detected a number of voiceless stops (or [-voice] [-continuant]) in several archaic attested languages (namely, Classical Greek, Latin and Sanskrit) that paralleled homorganic (i.e., the place of articulation) voiceless fricatives (or [-voice] [+continuant]), becoming what is known as the 1st Germanic Consonant Shift (separating Proto-Germanic from the others which retained voiceless stops of a common source): Verner's Law famously identified voluminous exceptions to Grimm's Law by associating them with one single sound-change rule; that is, in word-medial position, if the syllable succeeding voiceless stops affected by Grimm's Law received accent (stress and/or pitch), then the segments became voiced fricatives (or [+voice] [+continuant]). What has become commonly known among "generative" phonologists as a "feeding" relationship, Grimm's Law fed Verner's Law, through extending the natural class of original word-initial segments to word-medial segments, the latter of which were affected by Verner's Law [26]. Conventionally Bennett [27], a period of time for a Germanic Proto-Language to shift its accent to a syllable that was only word-initial must take "between several centuries to at least a millennium (p. 100), so, by this reckoning, the segments influenced by Grimm's Law and then by Verner's Law were approximately allophonic variants of each other at some stage later than Early Proto-Germanic; however, by Late Proto-Germanic, voiced fricatives created by the condition of Verner's Law underwent a diachronic process of fortition, or fricatives becoming stops [28].

Contemporary German and English present some remnants of this conjectural process (e.g., zieh-en v. zog-en, was v. were), but Old English, Old Icelandic, Old Norse and Old Saxon exhibit only the later-stage fortition procedure. Consequently, by the mid-1870's, Verner took only those attested forms as well as Sanskrit verbalmorphology paradigms in order to adduce one set of allo-forms (either -phonic or -morphic) of voiceless (qua Grimm) and voiced fricatives (in the environment of '...C...'). As for the reckoning of the timing of this operation, it cannot be definitely ascertained whether it was phonologically or morphologically conditioned, largely due to the degree to which these alternating voiceless $\sim$ voiced segments had been 'lexicalized' [26,28]. Whereas the contemporary English forms may be said to be morphologically conditioned by means of some combination of [be + PAST], they may also be deemed as thoroughly lexicalized (anomalous, or arbitrary); however, remnants from contemporary German allot enough parallels with attested historic Germanic forms to argue that they fit morphological conditioning (i.e., past-plural and pastparticiple paradigmatic forms). Regardless, they demonstrate morphophonemic (or grammatical) alternations despite exhibiting irregularities (which make them appear unsystematic) and were (qua Verner) not only unattested "by any external evidence" from Proto-Germanic forms but also bear the burden of verification "if we accept a [simplicity] criterion of descriptive economy... [and] reject... unordered rules" [24]. Likewise, Bever [29] makes a similar claim about sound segments, simplified into distinctive-feature natural classes with respect to "synchronic data... incidentally display[ing] morphophonemes and early rules identical with those of the parent language": He infers this idea from Bloomfield's insinuation of a synchronic Menomini morphophonemic subsystem 
closely approaching diachronic changes from the proto-language to the current one. Ordered rules, departing from a descriptive system that has been thus simplified, rise to a level of utility as well as apt precision due to observation ${ }^{3}$.

In terms of the previously mentioned diachronic change pertaining to Grimm and Verner, the latter must have succeeded the former as is suggested by the entire natural class of fricatives, those instigated by the 1st Germanic Consonant Shift (a.k.a., Grimm's Law) as well as one which not was included therein, the apparent lone member of the earliest Indo-European fricative, $-\boldsymbol{s}$.

The predictable change in voicing afforded to fricatives by Verner's Law affected the segment $-\boldsymbol{s}$ in the same way $(-\boldsymbol{s}>-\mathbf{z})$, as hinted in runic inscriptions as well as some in Gothic [30]. Later, it became evident in West and North Germanic that this voiced segment 'rhotacised' to $-\boldsymbol{r}$ and gave those varieties the imprint of verbal paradigms in their past-plurals and past-participles [27$29,31]$. Clearly, a procedure initiates a diachronic process but without synchronic timing to inform us of morphophonemics: However, some data attest that Germanic (e.g., Gothic and Old English) borrowings of the word Graeki from Latin employed a written symbol ' $\boldsymbol{k}$ '' word-initially and thus suggest that segments affected by Verner's Law (voiceless fricatives > voiced) still possessed allophonic variation (voiced fricatives $\sim$ voiced stops, a sign of synchronic variation owing to a fortition process) because Germanic varieties hadn't developed ' $\boldsymbol{g}$ - ' at least phonemically [32]. That is to say, based on the historical timing as per Grimm (voiceless stops > voiceless fricatives and voiced stops > voiceless stop), no voiced stops (< voiced fricatives) functioned phonemically for Latin Graeci (Bloomfield imagines that Germanic speakers heard k- from acoustic signals emitted by Latin speakers, Germanic having / $\gamma /$ as a fricative; 1933, p. 446). The closing step of the 1st Germanic Consonant Shift had yet to be terminated ( ${ }^{*}$ voiced fricatives > voiced stops $\mathbf{s}^{4}$ ] [33].

Germanic varieties preserved voiced stops at the time but only phonetically ( $<$ voiced fricatives in the environment of '...C...') and alternated with their senior and (perhaps) more basic forms. The timing of these sound-changes could never have been as absolutely precise because of the vagaries of historical distances as well as the relative absence of widespread written forms based on a singular orthographic fit. Moreover, the systems of spelling inherent to the varieties under discussion certainly lacked an apt sound-to-symbol correspondence in terms of orthoepy, so it would be problematic to decide if the writing were narrowly phonetic or broadly phonemic. Furthermore, reservations concerning two strata of sound (-emic and -etic) ensue therefrom, and, in relation to -emic qualities, disagreements over process (allegedly mental or purely historical) are brought to the fore. Such issues are urged to be laid by as nonessential and heed paid instead to sound-changes over time as undeniable (either mental processes or conditioning contexts); however, they remain linguistic, to an extent haphazard while at the same time amply systematic in yielding to an evaluation metric of relative simplicity expressing a structured sequential logic. Let's consent to Bailey [34] that synchronic (or static) statements about language $\mathrm{X}$ must be truly more dynamic due to its widespread variation, that structuralist descriptions were troubled by "generalized rules for morphophonic relationships" (and backed allomorphic variants, p. 18) and that diachronic patterning, despite its apparent dynamicity, takes the form of static accounts. The explanations afforded by linguistics were in fact not explanatory, in part because the method transformed words into numbers in order to gain the respect of others who believed in quantities. A drawback to this alternative wave framework [34] is that form is taken at face value, so language change is implausible as one form being organized as a 'monolect' of $\mathrm{X}$ in two ways (although a language learner in this model is allowed two different 'polylects' at the same time).

\section{Overt Identicalness vs. Covert Difference}

An provocative ambivalence regarding the difference between phonetics and phonemics is expressed in the work of Lees 1970, wherein he directs readers that he is not making a claim by his use of slant brackets (e.g., /x/) in order assert a phonemic stratum (p. 272, fn. 5); however, he states that these forms are understood there as 'underlying' and that any "phonetic character" of reconstructed proto-forms are nonetheless "superficial" (p. 271). It is easily understood that proto-forms (by their very nature) never possess the physical qualities of a narrow transcription of an actual phone and must therefore be only transcribed broadly, and Lees references forms throughout as being 'derived' and thus implies a process that is fashioned on a basic lexical item (i.e., a morphological operation, which appears morphophonemically regular and conservative, like a proto-form may trace through the ages). Tracing a problem of Turkish morphophonemics to a protoform that is still represented orthographically in Turkish as noninitial < - $\breve{g}$ - > (a form pronounced in Anatolian dialects of Turkish as voiced velar fricative [- $\boldsymbol{\gamma}-]$ but not in the environs of Istanbul), Lees proposes a slight alternation in the synchronic description of urban Turkish: Using a \pm system of pronunciations as well as a proto-form "ghost" consonant from proto-Turkic (namely non-initial /-g-/), the urban dialect of Turkish surrounding Istanbul at the time no longer inventoried the yumuşak-g (i.e., <- $\breve{g}$->) as part of its pronunciations and commenced to inventory four different long vowels that were indigenously born (i.e, not borrowed from Arabic words,

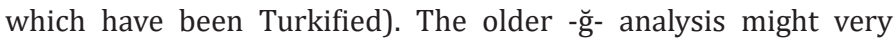
well be more efficient in terms of morphophonemic outputs and \pm decision-making of pronunciations than the newer -g- analysis, but the latter analysis benefits from being 'simpler' in terms of the morphophonemic selections chosen as outputs (despite several being unpredictable, or irregular). More thought-provoking about

\footnotetext{
${ }^{3}$ It should be noted that Bever informs his readers that Bloomfield kept his characteristic distance from mentalism and thus notated the source of Menomini morphophonemics as their conditioning environments, not as processes.

${ }^{4}$ means not yet attested in the word-initial postion of Germanic varieties, recognized as a series voiced spirants in Elliott (1959, pp. 15-18), including a version of a rhotacized-z- in word-medial position as per Verner's Law.
} 
the proposition put forward by Lees 1970 is that the addition of four long vowels increases the symmetry in the resonance cavity of the mouth, in that the short vowels / o † ö ü / include lengthened counterparts, just as / i e u a / have been lengthened by means of Arabic loanwords [35].

Language change in this instance concerns re-analysis of forms into a different pattern of organization based on the same input. One monolect mends identical forms that were organized by another monolect. As a result, pronunciations sound indistinguishable although categories of sound (i.e., phonemic bases) have been rearranged, "a distinct discontinuity in analysis rather than a gradual shift of the pronunciations involved" [3]; in other words, an array endures without change, yet its "internal structure" has in fact changed [31]. Startling though it may be, amending a grammatical system (sound-formation, word-formation and sentence-formation) seems as abrupt as Lightfoot's [36] proposal of the radical change from lexical open-class items to functional closed-class items in mid-16th century English, a kind of punctuated equilibrium, to use a biological metaphor. In this way, my discussion returns (perhaps equally abruptly) to anthropology and archaeology: It is fitting when synchronic data (i.e., a static, descriptive grammar) can be verified impartially through the application of linguistically external evidence given by reconstructed diachronic data, and such data are found, pointedly, through the Word (and, on occasion) in the dirt. Neutrality has been articulated regarding how long-term storage is maintained though the random nature of a mental dictionary and an orderly kernel of the grammar of X has been affirmed; likewise, the discussion is neutral concerning -etic and -emic strata of linguistic expression, but it has been too strongly suggested, plausibly, that only one stratum is not enough yet more than one stratum is far too many. A key to simplicity and to order is quantification but only with respect to language, not to culture, and the development of language and culture proceed hand-in-hand but without singular uniformity: Language has only structure yet interacts with process (via cognition) and production/perception (via communication). Communication is a zone querying across disciplines $[37,38]$.

\section{Brief Closing Remarks}

More often than not, the Word is found in the air (or the egressive pulmonic airstream), and the work of the linguist occasionally is unearthing what in human languages is remarkable; for example, "[e]ven more weird is the fact that the new analysis [by Lees] appears to restore Proto-Turkic*/g/ (and also Altaic long vowels, though only before /g/") (1970, p. 279). Observing behavior directly, devoid of any biases in its description yet full in its achievement, is the work and Word of a linguist, like it was for Darwin as it was for Bacon, natural scientists.

One can only muse upon why or how most sounds/gestures have one or several meanings, but few if any feel gratified, as Mead (2006) described people's reactions in her dream about Boas, at a sense of wonder for organic Life ("only wonder comprehends anything": Gregory of Nyssa).

\section{References}

1. Ortman SG (2000) Conceptual metaphor in the archaeological record: Methods and an example from the American Southwest. American Antiquity 65(4): 613-645.

2. Vermeule E (1996) Archaeology and philology: The Dirt and the Word. Transactions of the American Philological Society 126: 1-10.

3. Lees RB A morphophonemic problem in Turkish. In: Akin A, Goldberg A, Myers G, Stewart J. Language and Behavior. The Hague, Mouton, Netherlands pp. 271-279.

4. Ellis R (1994) The study of second language acquisition ( $1^{\text {st }}$ Edn.) Oxford University Press, Oxford, UK.

5. Kariloff Smith A (1986) Stage/structure versus phase/process in modelling linguistic and cognitive development. In: I Levin (Eds.) Stage and structure: Re-opening the debate. Ablex, New Jersey, USA.

6. Saussure Fd (1959) Course in general linguistics. In: Bally B, Sechehaye A (Eds.), McGraw-Hill, New York, USA.

7. Piaget J (1926) La representation du monde a chez l'enfant. Presses Universitairesde France, Paris, France.

8. Romaine S (1995) Bilingualism. Blackwell, Oxford, England.

9. Griffiths P (1986) Early vocabulary. In: Fletcher P, Garman M (Eds.), Language acquisition ( $2^{\text {nd }}$ Edn.). Cambridge University Press, Cambrigde, UK.

10. Carey S (1978) The child as word learner. In: Halle M, Bresnan J, Miller GA (Eds.), Linguistic Theory and Psychological Reality. The MIT Press, Cambridge, Massachusetts, USA.

11. Bowerman M (1994) Learning a semantic system: What role do cognitive predispositions play? In: Bloom P (Ed.), Language acquisition: Core readings. The MIT Press, Cambridge, Massachusetts, USA.

12. Goodluck H (1991) Language Acquisition. Blackwell, Oxford, England.

13. Maquet J (1970) Review of the book the raw and the cooked: Introduction to a science of mythology by C Levi-Strauss. Technology and culture 11(4): 613-615.

14. Strevens P (1991) Applied linguistics: On overview. In: Grabe W, Kaplan R (Eds.) Introduction to applied linguistics p. 12-30.

15. Penzl H (1972) Methods of comparative Germanic linguistics. In: Fv Coetsem, HL Kufner (Eds.), Toward a grammar of proto-Germanic. Max Niemayer Verlag, Tübingen, Germany p.1-42.

16. Lehmann WP (1972) Descriptive linguistics: An introduction. Random House, New York, USA.

17. Siertsema B (1965) A study of glossematics. The Hague: Martinus Nijloff, Netherlands.

18. Hockett C (1968) Review of the book Outline of stratificational grammar, by S Lamb. International Journal of American Linguistics 34(2): 145153.

19. Culler J (1976) Ferdinand de Saussure. Penguin, New York, USA.

20. Hockett C (1954) Two Models of Grammatical Description. Word 10(23): $210-234$.

21. Trubetzkoy NS (1939) Grundzüge der phonologie. Göttingen: Vendenhoeck and Ruprecht.

\footnotetext{
${ }^{5}$ represents an unattested form that has been reconstructed in only non-initial positions.

${ }^{6} \mathrm{~A}$ proposed language family (Proto-Turkic being one sub-family) exhibiting numerous non-genetic characteristics.

${ }^{7}$ Türkmen belongs to a Proto-Turkic family (as does Turkish) retaining contrastive distribution in short/long vowels (Lees, 1970, p. 273).
} 
22. Lehmann WP (1972) A reader in $19^{\text {th }}$ century historical Indo-European linguistics. Indiana University Press, Bloomington, USA.

23. Hock HH (1986) Principles of historical linguistics. Mouton de Gruyter, New York, USA.

24. Halle M (1961) On the role of simplicity in linguistic descriptions. In: Jakobson R, Structure and language and its mathematical aspects. Providence RI: Proceedings from $12^{\text {th }}$ symposium in applied mathematics p. 89-94.

25. Halle M, Clements GN (1983) Problem book in phonology. Cambridge, MIT Press, London, UK.

26. Coetsem Fv (1972) Proto-Germanic morphophomenics. In: Fv Coetsem, HL Kufner (Eds.), Toward a grammar of proto-Germanic. Max Niemayer Verlag, Tübingen, Germany pp. 175-209.

27. Bennett WB (1972) Prosodic features in proto-Germanic. In: Fv Coetsem, HL Kufner (Eds.), Toward a gammar of proto-Germanic. Max Niemayer Verlag, Tübingen, Germany pp.175-209.

28. King RD (1969) Historical linguistics and generative grammar. PrenticeHall, New Jersey, USA.

29. Bever TG (1963) Theoretical implications of Bloomfield's Menomini Morphophonemics. In: Fought F (Ed.), Leanard Bloomfield: Critical assessments of leading linguists. Routledge, New York, USA pp. 13-19.
30. Elliott RWV (1959) Runes. St. Martin's, New York, USA.

31. Campbell L (1998) Historical linguistics. The MIT Press, Cambridge, Massachusetts, USA.

32. Moulton WG (1972) The proto-Germanic non-syllabics. In: Fv Coetsem, HL Kufner (Eds.), Toward a grammar of proto-Germanic. Max Niemayer Verlag, Tübingen, Germany pp.141-173.

33. Bloomfield L Language. Holt Rinehart and Winston, Chicago, USA.

34. Bailey C JN (1973) Variation and linguistic theory. Center for applied linguistics, Arlington, Virgenia, USA.

35. Antttila R (1972) An introduction to historical and comparative linguistics. Macmillan, New York, USA.

36. Lightfoot D (1979) Principle of diachronic syntax. Cambridge University Press, Cambridge, Massachusetts, USA.

37. Clevenger Jr T (1960) Some characteristics of the study communication in 1960. The journal of communation 10(4): 163-173.

38. Caffrey MM, Francis PA (2006) Margaret Mead's letters. Basic Books, Cambridge, Massachusetts, USA.

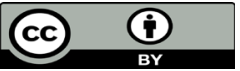

This work is licensed under Creative Commons Attribution 4.0 License

To Submit Your Article Click Here:

Submit Article

DOI: $10.32474 / J A A S .2020 .01 .000120$

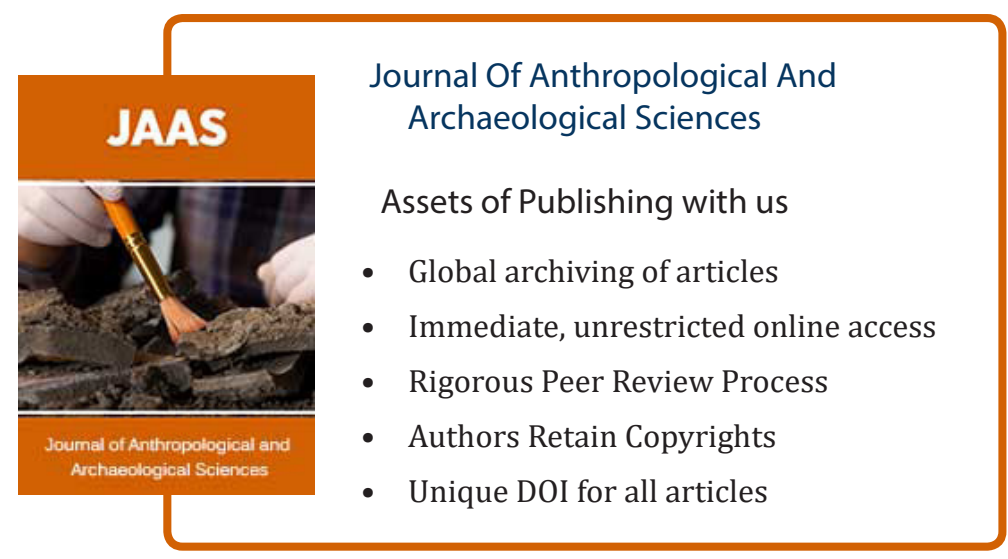

Citation: Tully J Thibeau. Populations and Excavations; Strata and Data: A linguist Muses upon Anthropology and Archaeology. J Anthro 